\title{
Hypoxia and hypotension in patients intubated by physician staffed helicopter emergency medical services - a prospective observational multi-centre study
}

Geir Arne Sunde ${ }^{1,2,3,17^{*}}$ (D) Mårten Sandberg ${ }^{4,5}$, Richard Lyon ${ }^{6,7}$, Knut Fredriksen $^{8,9}$, Brian Burns ${ }^{10,11}$, Karl Ove Hufthammer ${ }^{12}$, Jo Røislien ${ }^{1,3}$, Akos Soti ${ }^{13}$, Helena Jäntti ${ }^{14}$, David Lockey ${ }^{3,15}$, Jon-Kenneth Heltne ${ }^{2,16}$ and Stephen J. M. Sollid ${ }^{1,3,4}$

\begin{abstract}
Background: The effective treatment of airway compromise in trauma and non-trauma patients is important. Hypoxia and hypotension are predictors of negative patient outcomes and increased mortality, and may be important quality indicators of care provided by emergency medical services. Excluding cardiac arrests, critical trauma and non-trauma patients remain the two major groups to which helicopter emergency medical services (HEMS) are dispatched. Several studies describe the impact of pre-hospital hypoxia or hypotension on trauma patients, but few studies compare this in trauma and non-trauma patients. The primary aim was to describe the incidence of pre-hospital hypoxia and hypotension in the two groups receiving pre-hospital tracheal intubation (TI) by physician-staffed HEMS.

Methods: Data were collected prospectively over a 12-month period, using a uniform Utstein-style airway template. Twenty-one physician-staffed HEMS in Europe and Australia participated. We compared peripheral oxygen saturation and systolic blood pressure before and after definitive airway management. Data were analysed using Cochran-Mantel-Haenszel methods and mixed-effects models.

Results: Eight hundred forty three trauma patients and 422 non-trauma patients receiving pre-hospital $\mathrm{Tl}$ were included. Non-trauma patients had significantly lower predicted mean pre-intervention $\mathrm{SpO}_{2} \mathrm{Compared}$ to trauma patients. Post-intervention and admission $\mathrm{SpO}_{2}$ for the two groups were comparable. However, 3\% in both groups were still hypoxic at admission. For hypotension, the differences between the groups were less prominent. However, $9 \%$ of trauma and $10 \%$ of non-trauma patients were still hypotensive at admission. There was no difference in short-term survival between trauma (97\%) and non-trauma patients (95\%). Decreased level of consciousness was the most frequent indication for $\mathrm{Tl}$, and was associated with increased survival to hospital (COR 2.8; 95\% Cl: 1.4-5.4).

(Continued on next page)
\end{abstract}

\footnotetext{
* Correspondence: geir.arne.sunde@norskluftambulanse.no

${ }^{1}$ Norwegian Air Ambulance Foundation, Drøbak, Norway

${ }^{2}$ Department of Anaesthesia and Intensive Care, Haukeland University

Hospital, Bergen, Norway

Full list of author information is available at the end of the article
} 
(Continued from previous page)

Conclusions: Our results showed that non-trauma patients had a higher incidence of hypoxia before TI than trauma patients, but few were hypoxic at admission. The difference for hypotension was less prominent, but one in ten patients were still hypotensive at admission. Further investigations are needed to identify reversible causes that may be corrected to improve haemodynamics in the pre-hospital setting. We found high survival rates to hospital in both groups, suggesting that physician-staffed HEMS provide high-quality emergency airway management in trauma and non-trauma patients.

Trial registration: Clinicaltrials.gov Identifier: NCT01502111. Registered 22 Desember 2011.

Keywords: Physician staffed HEMS, Airway management, Intubation, Air ambulance, Helicopter emergency medical services, Advanced trauma life support, Critical care

\section{Background}

Pre-hospital advanced airway management including tracheal intubation (TI) has high priority in the management of critically ill patients [1-3]. Drug-assisted rapid sequence intubation (RSI) is the definitive method of securing the airways of patients who are unable to maintain patent airways or adequate ventilation [2]. However, TI in the prehospital setting may be challenging, with sub-optimal working conditions for critical care providers [4]. Several studies report a high incidence of unanticipated difficult airways, first TI attempt failures and complications during pre-hospital advanced airway management, comparable to emergency airway management outside the operating room [5-8]. Critically ill patients may be susceptible to hypoxia and hypotension in conjunction with emergency anaesthesia and airway management [9-11]. The full range of optimal emergency airway management requires an experienced and trained provider to manage it, and hospital-level care to patients in the field is often provided by physician-staffed helicopter emergency medical services (HEMS) [12-14].

Pre-hospital hypoxia and hypotension are predictors of negative patient outcomes and increased in-hospital mortality in non-cardiac arrest patients, and avoidance or mitigation of hypoxia and hypotension may be considered important measures of quality of care provided by the emergency medical services (EMS) [15-17]. Sadly, core data on physiological responses and how they relate to pre-hospital TI are inconsistently reported. Standardised data from pre-hospital airway management could improve our knowledge about the challenges of hypoxia and hypotension in TI [18-20].

The target group of this multi-centre study were noncardiac arrest patients requiring pre-hospital TI by physician-staffed HEMS. By excluding out-of-hospital cardiac arrests, critical trauma and non-trauma patients are the major groups to which HEMS are dispatched [21]. Several studies describe the impact of pre-hospital hypoxia or hypotension on trauma patients but few studies compare this to the impact hypoxia and hypotension has on non-trauma patients needing pre-hospital TI. This knowledge could be important for how the two groups are handled in pre-hospital care $[15,22]$. The primary aim of our study was to describe the incidence of pre-hospital hypoxia and hypotension in the two groups. Secondarily, we wanted to assess whether survival to hospital differed between trauma and nontrauma patients.

\section{Methods}

\section{Study design and setting}

This prospective multi-centre study collected uniform data on advanced pre-hospital airway management from 21 HEMS in Australia, England, Finland, Hungary, Norway and Switzerland, to analyse differences between trauma and non-trauma patients requiring TI in the field. To include the full range of resuscitative interventions on-scene, only physician-staffed HEMS participated. Necessary ethical and institutional approvals were acquired prior to patient enrolment.

\section{Participants}

Trauma and non-trauma patients requiring pre-hospital TI on primary missions were included. Primary out-ofhospital cardiac arrests were excluded. Airway management and RSI protocols were part of local standard operating procedures. Service-specific anaesthetic agents, sedatives, analgesics and neuromuscular blocking agents were used to facilitate TI.

\section{Data collection}

Data collections lasted for 1 year for the majority of centres, commencing on the 1st of January 2012 for the majority of centres and concluded on the 15th of March 2013 for the last centre. Two centres, Kent Surrey Sussex HEMS (England) and REGA-Basel (Switzerland), participated for 9 and 6 months, respectively. Prospective airway data were collected according to dataset definitions described in the Utstein style template [23]. Survival data was available only for the pre-hospital 
phase, as in-hospital follow-up was beyond the scope of this study. Data regarding airway management success rates and complications have previously been published [24].

\section{Variables}

Patient demographics were described by category (trauma or non-trauma), age, sex, and indications for TI. Burns and strangulation were classified as trauma in the template, while drownings and asphyxia were classified as non-trauma. We compared lowest oxygen saturation $\left(\mathrm{SpO}_{2}\right)$ and systolic blood pressure (SBP) before and after completion of TI as described in the airway template. An intubation attempt was defined as attempted laryngoscopy with the intent to intubate. Hypoxia was defined as $\mathrm{SpO}_{2}<90 \%$ and hypotension as $\mathrm{SBP}<90 \mathrm{mmHg}$. $\mathrm{S}_{\mathrm{P}} \mathrm{O}_{2}$ and $\mathrm{SBP}$ were measured as first value recorded on scene and first value recorded after finalised airway management. Glasgow Coma Score (GCS) and pre-intervention comorbidity (American Society of Anesthesiologists Physical Status (ASA-PS)) were recorded. The variables are defined in the airway template paper [23].

\section{Statistical analysis}

Categorical demographical variables are presented as counts and percentages. The multi-centre nature of the study introduces an internal clustering in the data, and the statistical methods that take this into account have thus been applied [25]. We were mainly interested in the intra-site association between the recorded variables. For binary outcomes we applied Cochran-Mantel-Haenszel methods to estimate conditional odds ratios (cOR) and corresponding 95\% confidence intervals (CIs), while for continuous outcomes we used mixed-effects models with random intercepts for sites for single-value outcomes and for sites and patients for longitudinal data. Results from these models are presented as predicted means. The predicted mean is an estimate of the mean for a patient from a 'typical' site, i.e. with the random effect(s) set to zero, and can roughly be interpreted as a 'mean of means' estimate. Marginal estimates are also presented. The amount of missing data for the main outcomes was generally low, and we have therefore performed complete-case analyses throughout.

We used IBM SPSS Statistics version 21.0 (IBM Corp., Armonk, New York) for storing and preparing the data for statistical analysis and $\mathrm{R}$ version 3.2.1 and 3.2.3 ( $\mathrm{R}$ Foundation for Statistical Computing, Vienna, Austria) for performing the analyses [26]. For fitting the mixedeffects models, we used the $\mathrm{R}$ packages 'nlme' version 3.1-123 and 'Ime4' version 1.1-10.

\section{Results}

\section{Demographics}

Overall, 2327 (16\%) of patients attended required advanced airway interventions during the study period. We included 1265 patients receiving pre-hospital TI in the analysis. Of these, 843 were trauma patients and 422 non-trauma patients. Patients handled with bag-valvemask ventilation (BVM), supraglottic airway devices (SAD) or continous positive airway pressure (CPAP) instead of TI and patients with missing data relative to airway management, short-term survival status or trauma categories were excluded from the analysis (Fig. 1). Patient characteristics, indication for TI and number of attempts are summarised in Table 1. Decreased level of consciousness was the most frequent indication recorded in both trauma (61\%) and nontrauma patients (69\%). Emergency surgical airway was done in two trauma patients $(0.2 \%)$, one after failed primary TI and one was a primary surgical airway. The proportion of males did not differ significantly between trauma and non-trauma patients (cOR: 1.27; 95\% CI: 0.94-1.73). There was a large difference in predicted mean age between trauma and non-trauma patients (43 and 54 years; CI of difference: 8-14 years). Non-trauma patients had a predicted mean initial GCS of 2.1 points lower (95\% CI: $1.6-2.6)$ than trauma patients (predicted means 5.6 and 7.6, respectively).

\section{Oxygen saturation}

Non-trauma patients had significantly lower preintervention $\mathrm{SpO}_{2}(p<0.001)$ and post-intervention $\mathrm{SpO}_{2}(p=0.001)$ than trauma patients, predicted means 89 vs. $94 \%$, and 96 vs. $97 \%$, respectively (Fig. 2). Admissions mean $\mathrm{SpO}_{2}$ was $98 \%$ for both groups. In both groups, 3\% of patients were still hypoxic at admission (Table 2). Rates of hypoxia and hypotension in patients with decreased level of consciousness are presented in Table 3.

\section{Systolic blood pressure}

There was a significant difference in predicted mean pre-intervention SBP between non-trauma and trauma patients $(p=0.002)$, but not for SBP at post-intervention or at admission (Fig. 2). For non-trauma patients, there was a significant decrease $(p<0.001)$ in SBP during the airway intervention from a pre-intervention (predicted mean) SBP of $135 \mathrm{mmHg}$ to post-intervention SBP $120 \mathrm{mmHg}$, and a subsequent significant increase to hospital admission SBP of $124 \mathrm{mmHg}$. For trauma patients, there was a significant $(p<0.001)$ decrease in SBP during the airway interventions from $127 \mathrm{mmHg}$ to $121 \mathrm{mmHg}$, but post-intervention SBP was equal to the 


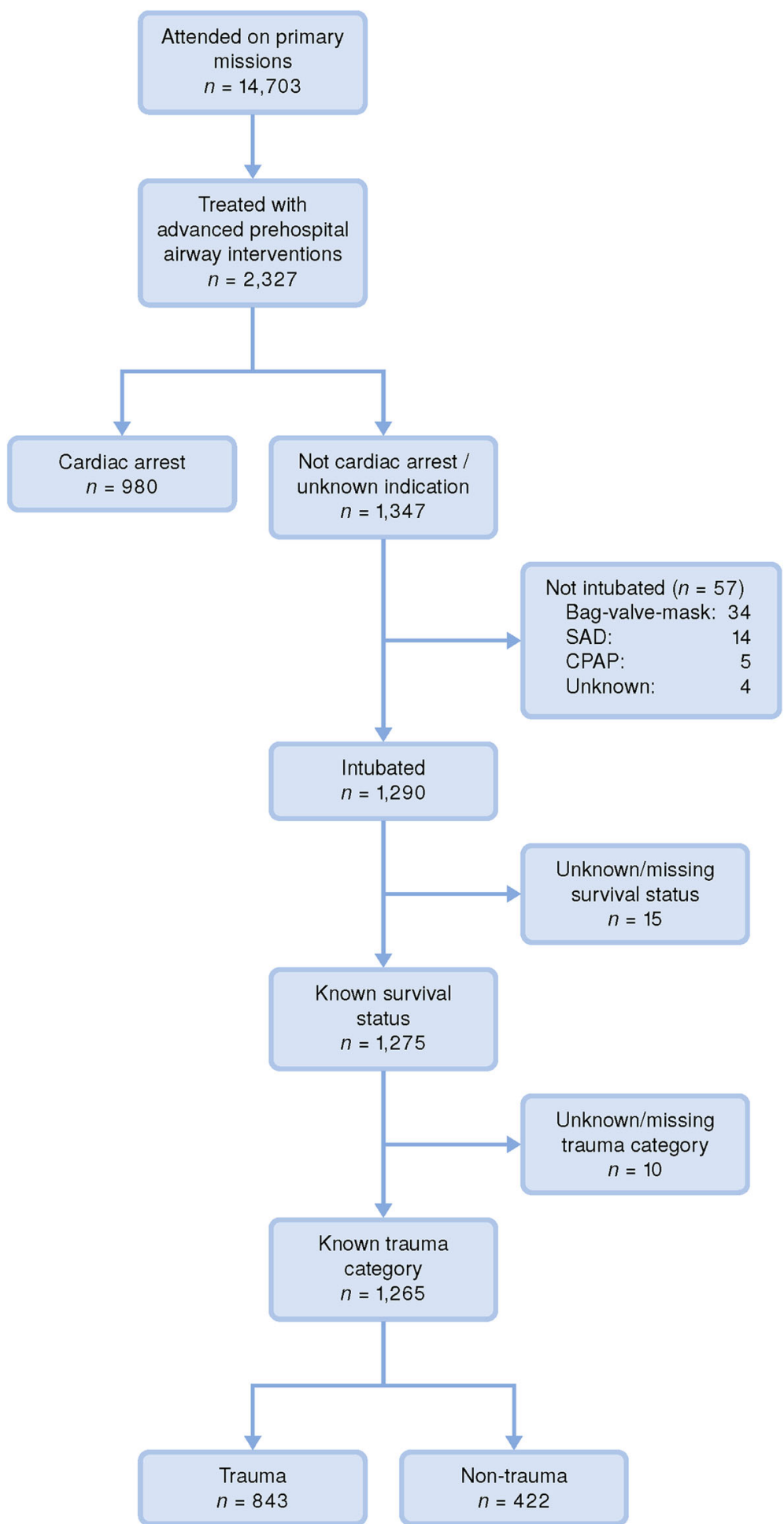

Fig. 1 Study population flow chart. Flow chart of study population. One thousand two hundred sixty five non-cardiac arrest patients that received pre-hospital tracheal intubation were included

admission SBP of $121 \mathrm{mmHg}$. Nine percent of trauma patients and $10 \%$ of non-trauma patients were still hypotensive at admission (Table 2).

\section{Short-term survival}

Overall, 97\% of patients survived to hospital admission. There was no difference in short-term survival between 
Table 1 Marginal/crude patient characteristics

\begin{tabular}{|c|c|c|c|c|c|c|c|}
\hline \multirow[t]{2}{*}{ Patient category } & \multicolumn{2}{|c|}{ Trauma } & \multicolumn{2}{|c|}{ Non-trauma } & \multicolumn{2}{|c|}{ All patients } & \multirow[t]{2}{*}{$P$-value } \\
\hline & $n$ & $\%$ & $n$ & $\%$ & $n$ & $\%$ & \\
\hline Patients & 843 & $100 \%$ & 422 & $100 \%$ & 1265 & $100 \%$ & \\
\hline Age & & & & & & & $<0.001$ \\
\hline $0-5$ years & 19 & $2 \%$ & 22 & $5 \%$ & 41 & $3 \%$ & \\
\hline $6-14$ years & 36 & $4 \%$ & 9 & $2 \%$ & 45 & $4 \%$ & \\
\hline $15-29$ years & 227 & $27 \%$ & 38 & $9 \%$ & 265 & $21 \%$ & \\
\hline $30-49$ years & 276 & $33 \%$ & 83 & $20 \%$ & 359 & $29 \%$ & \\
\hline 50-69 years & 185 & $22 \%$ & 151 & $37 \%$ & 336 & $27 \%$ & \\
\hline$>70$ years & 83 & $10 \%$ & 109 & $26 \%$ & 192 & $16 \%$ & \\
\hline Missing data & 17 & $2 \%$ & 10 & $2 \%$ & 27 & $2 \%$ & \\
\hline Sex & & & & & & & $<0.001$ \\
\hline Male & 622 & $74 \%$ & 266 & $64 \%$ & 888 & $70 \%$ & \\
\hline Missing data & 1 & $0 \%$ & 3 & $1 \%$ & 4 & $0 \%$ & \\
\hline Comorbidity (ASA-PS) & & & & & & & $<0.001$ \\
\hline ASA class 1 & 500 & $67 \%$ & 100 & $26 \%$ & 600 & $53 \%$ & \\
\hline ASA class 2 & 188 & $25 \%$ & 146 & $38 \%$ & 334 & $29 \%$ & \\
\hline ASA class 3 & 58 & $8 \%$ & 120 & $31 \%$ & 178 & $16 \%$ & \\
\hline ASA class 4 & 4 & $1 \%$ & 19 & $5 \%$ & 23 & $2 \%$ & \\
\hline ASA class 5 & 0 & $0 \%$ & 4 & $1 \%$ & 4 & $0 \%$ & \\
\hline Missing data & 90 & $11 \%$ & 29 & $7 \%$ & 119 & $9 \%$ & \\
\hline Indication for pre-hospital TI & & & & & & & $<0.001$ \\
\hline Decreased consciousness & 510 & $61 \%$ & 277 & $69 \%$ & 787 & $64 \%$ & \\
\hline Ineffective ventilation & 69 & $8 \%$ & 58 & $14 \%$ & 127 & $10 \%$ & \\
\hline Combative or uncooperative & 93 & $11 \%$ & 9 & $2 \%$ & 102 & $8 \%$ & \\
\hline Impending airway obstruction & 68 & $8 \%$ & 12 & $3 \%$ & 80 & $6 \%$ & \\
\hline Hypoxia & 26 & $3 \%$ & 25 & $6 \%$ & 51 & $4 \%$ & \\
\hline Relief of pain or distress & 44 & $5 \%$ & 4 & $1 \%$ & 48 & $4 \%$ & \\
\hline Existing airway obstruction & 13 & $2 \%$ & 12 & $3 \%$ & 25 & $2 \%$ & \\
\hline Other & 8 & $1 \%$ & 6 & $1 \%$ & 14 & $1 \%$ & \\
\hline Missing data & 12 & $1 \%$ & 19 & $5 \%$ & 31 & $2 \%$ & \\
\hline Attempts at pre-hospital TI & & & & & & & $<0.001$ \\
\hline One attempt & 772 & $92 \%$ & 360 & $86 \%$ & 1132 & $90 \%$ & \\
\hline Multiple attempts & 68 & $8 \%$ & 60 & $14 \%$ & 128 & $10 \%$ & \\
\hline Missing data & 3 & $0 \%$ & 2 & $0 \%$ & 5 & $0 \%$ & \\
\hline
\end{tabular}

Characteristics of patients requiring pre-hospital TI by physician-staffed HEMS, TI Tracheal intubation, HEMS Helicopter emergency medical services, ASA-PS American Society of Anesthesiologists Physical Status. All percentages except for the 'Missing data' rows are calculated based on the non-missing data

trauma patients $(97 \%)$ and non-trauma patients $(95 \%)$ $(\mathrm{cOR}=0.78 ; 95 \% \mathrm{CI}: 0.34-1.68 ; p=0.56)$. The large group intubated for decreased level of consciousness showed a more positive association with survival to hospital $(\mathrm{cOR}=2.8 ; 95 \% \mathrm{CI}: 1.4-5.4 ; p<0.001)$ than other indications for TI combined (Table 1). This effect was strong for non-trauma patients $(\mathrm{cOR}=10.7 ; 95 \% \mathrm{CI}$ : $2.7-42.1 ; p=0.001)$ but not present for trauma patients $(\mathrm{cOR}=1.2 ; 95 \% \mathrm{CI}: 0.49-2.9 ; p=0.69)$.

\section{Discussion}

Main findings

Non-trauma patients had a significantly higher incidence of hypoxia before TI than trauma patients. Postintervention and admission $\mathrm{SpO}_{2}$ for the two groups were comparable, and $3 \%$ in both groups were still hypoxic at admission. For hypotension, the differences between the groups were less prominent, but one in ten trauma and non-trauma patients were still hypotensive 


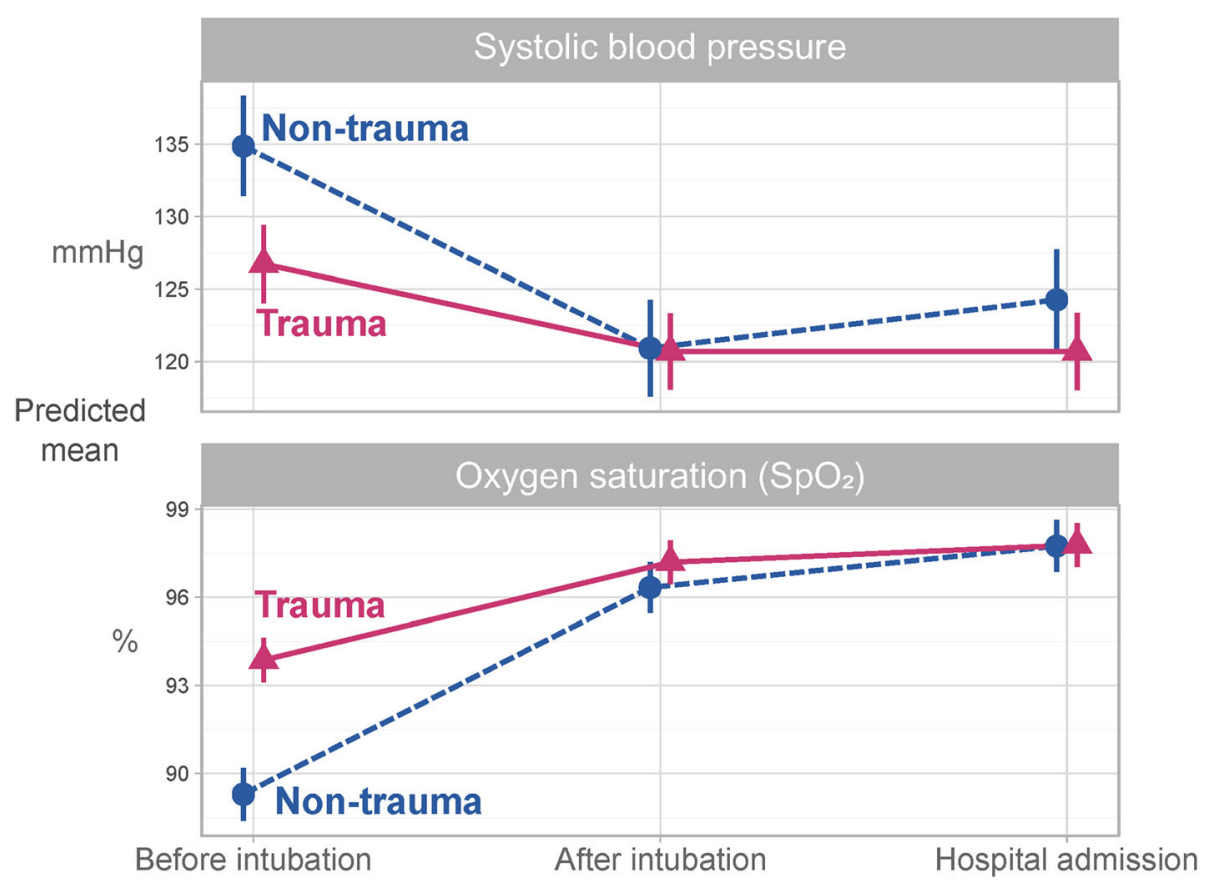

Fig. 2 Patient vitals across airway intervention. Predicted means for patients $\mathrm{SBP}$ and $\mathrm{S}_{\mathrm{p}} \mathrm{O}_{2}$ across airway intervention for trauma and non-trauma patients, based on linear mixed-effects models with time, trauma category and their interaction as fixed effects and random intercepts for patients and HEMS. Vertical lines show 95\% pointwise confidence intervals. Non-trauma patients had a significantly lower mean $\mathrm{SpO}_{2}$, and higher mean SBP, before TI compared to trauma patients. Post-intervention and admission values for the two groups showed little difference. SBP: systolic blood pressure. Sp $\mathrm{O}_{2}$ : oxygen saturation. HEMS: helicopter emergency medical services

at admission. There was no difference in survival to hospital between the groups studied, but patients intubated due to decreased level of consciousness showed a positive association with short-term survival than patients with other indications for TI.

\section{Vital signs and emergency anaesthesia}

Vital signs are commonly used for initial assessment and triage of patients, both in the pre-hospital setting and emergency department [27]. Hypoxia and hypotension in the field are predictors of increased in-hospital mortality, but patients' vital signs across airway interventions are infrequently reported [16, 18, 28]. Physiological variables like SBP and $\mathrm{SpO}_{2}$ may represent indications for $\mathrm{TI}$, but they are also markers of success or complications following airway intervention and the level of critical care provided in the field [23, 29]. As the pathophysiology behind pre-hospital hypoxia and hypotension are

Table 2 Hypotension and hypoxia rates before and after airway intervention

\begin{tabular}{|c|c|c|c|c|c|c|c|c|c|}
\hline \multirow[t]{2}{*}{ Patients } & \multicolumn{2}{|c|}{ Trauma } & \multicolumn{2}{|c|}{ Non-trauma } & \multirow{2}{*}{$\begin{array}{l}P \text {-value } \\
-\end{array}$} & \multicolumn{2}{|c|}{ All patients } & \multicolumn{2}{|c|}{ Missing data } \\
\hline & 843 & $100 \%$ & 442 & $100 \%$ & & 1265 & $100 \%$ & - & - \\
\hline \multicolumn{10}{|l|}{ Hypoxiab $^{\text {b }}$} \\
\hline Pre-intervention & 126 & $18 \%$ & 114 & $33 \%$ & 0.01 & 240 & $23 \%$ & 212 & $17 \%$ \\
\hline Post-intervention & 40 & $5 \%$ & 31 & $8 \%$ & 0.17 & 71 & $6 \%$ & 106 & $8 \%$ \\
\hline Admission to hospital & 23 & $3 \%$ & 12 & $3 \%$ & 0.30 & 35 & $3 \%$ & 164 & $13 \%$ \\
\hline \multicolumn{10}{|l|}{ Hypotension ${ }^{c}$} \\
\hline Pre-intervention & 87 & $12 \%$ & 56 & $15 \%$ & 0.83 & 143 & $13 \%$ & 157 & $12 \%$ \\
\hline Post-intervention & 100 & $13 \%$ & 63 & $16 \%$ & 0.97 & 163 & $14 \%$ & 77 & $6 \%$ \\
\hline Admission to hospital & 68 & $9 \%$ & 37 & $10 \%$ & 0.12 & 105 & $9 \%$ & 148 & $12 \%$ \\
\hline
\end{tabular}

The reported rates are marginal rates

a Based on Cochran-Mantel-Haenszel chi-squared tests

${ }^{b} \mathrm{Hypoxia}$ was defined as oxygen saturation $\left(\mathrm{SpO}_{2}\right)<90 \%$

${ }^{c}$ Hypotension was defined as systolic blood pressure (SBP) $<90 \mathrm{mmHg}$ 
Table 3 Hypotension and hypoxia in patients with decreased level of consciousness

\begin{tabular}{|c|c|c|c|c|c|c|c|c|c|}
\hline \multirow[t]{2}{*}{ Patients } & \multicolumn{2}{|c|}{ Trauma } & \multicolumn{2}{|c|}{ Non-trauma } & \multirow{2}{*}{$\begin{array}{l}P \text {-value }{ }^{a} \\
-\end{array}$} & \multicolumn{2}{|c|}{ All patients } & \multicolumn{2}{|c|}{ Missing data } \\
\hline & 510 & $100 \%$ & 277 & $100 \%$ & & 787 & $100 \%$ & - & - \\
\hline \multicolumn{10}{|l|}{ Hypoxia $^{\text {b }}$} \\
\hline Pre-intervention & 63 & $15 \%$ & 55 & $23 \%$ & 0.23 & 118 & $18 \%$ & 118 & $15 \%$ \\
\hline Post-intervention & 17 & $4 \%$ & 13 & $5 \%$ & 0.37 & 30 & $4 \%$ & 56 & $7 \%$ \\
\hline Admission to hospital & 7 & $2 \%$ & 3 & $1 \%$ & 0.19 & 10 & $1 \%$ & 83 & $11 \%$ \\
\hline \multicolumn{10}{|l|}{ Hypotension ${ }^{c}$} \\
\hline Pre-intervention & 48 & $11 \%$ & 27 & $11 \%$ & 0.63 & 75 & $11 \%$ & 88 & $11 \%$ \\
\hline Post-intervention & 55 & $11 \%$ & 28 & $11 \%$ & 0.44 & 83 & $11 \%$ & 38 & $5 \%$ \\
\hline Admission to hospital & 34 & $7 \%$ & 17 & $7 \%$ & 0.20 & 51 & $7 \%$ & 77 & $10 \%$ \\
\hline
\end{tabular}

The reported rates are marginal rates

aased on Cochran-Mantel-Haenszel chi-squared tests

${ }^{b}$ Hypoxia was defined as oxygen saturation $\left(\mathrm{SpO}_{2}\right)<90 \%$

'Hypotension was defined as systolic blood pressure (SBP) $<90 \mathrm{mmHg}$

diverse, it is important that these vital signs are interpreted along with other clinical variables and the mechanism of injury or illness [27, 30]. Resuscitative interventions are often initiated before the cause of hypotension or hypoxia is clearly identified [31]. The objectives of emergency anaesthesia and TI is to secure oxygenation and ventilation, but also to avoid secondary insults caused by hypoxia and hypotension to vital organs [1]. After initial resuscitation and stabilisation of the patient on-scene, pre-hospital critical care also includes a transition from anaesthesia to mobile critical care during the evacuation and transport of the patient to hospital. Although proper regular monitoring of vital signs is a priority in patients receiving emergency anaesthesia in the field, careful preparation and adequate monitoring to avoid complications can be more difficult in a pre-hospital setting than in a hospital $[1,32]$.

\section{Hypoxia}

One in three non-trauma patients in our study presented with pre-intervention hypoxia, and this was significantly more frequent than for trauma patients. However, in both groups $3 \%$ remained hypoxic at hospital admission after pre-hospital TI. Similar results have been reported from other physician-manned EMS [33, 34]. As desaturation may develop more rapidly in critically ill patients receiving emergency anaesthesia, strategies to improve preoxygenation during RSI in the field, like apnoeic oxygenation, may be important to reduce hypoxia in these patients $[9,22,35]$. We have previously published data showing a non-linear association between the patient's age and the TI failure risk, with the highest risk for middle-aged patients and significantly lower risk for both younger and older patients [24]. Another study demonstrated significantly higher age among all patients experiencing desaturation during pre-hospital RSI, and also showed that the duration of hypoxia was significantly longer in non-trauma patients compared to trauma patients [22]. Physician-staffed EMS provide TI success rates of close to $100 \%$ with very high first pass success rates and robust RSI procedures that are effective in preventing or correcting hypoxia. Highest quality airway management can be provided before arrival in hospital $[18,36,37]$.

\section{Hypotension}

Hypotension in trauma patients is often due to hypovolemia from blood loss, while non-traumatic hypotension may be due to a variety of causes, including hypovolemic, cardiogenic, septic or neurological factors, and it may be difficult to determine the exact cause of nontraumatic hypotension in the pre-hospital setting $[16,31]$. In-hospital mortality rates in non-trauma patients after pre-hospital hypotension have also shown to be high across all age groups [16, 17]. Furthermore, sustained hypotension or shock in trauma and non-trauma patients correlates with higher in-hospital mortality [17, 38]. Although clinical thresholds for hypotension related to patient outcomes, e.g. $90 \mathrm{mmHg}$ for severe traumatic brain injury (TBI), has been suggested, recent studies indicate a possible linear association between pre-hospital SBP and the probability of death, suggesting that using thresholds might not be so meaningful [15, 30, 39]. Nonetheless, a limit of $90 \mathrm{mmHg}$ to indicate hypotension was used in our study, in agreement with clinical guidelines and other systems handling critical ill patients $[16,31,40]$. The reductions in SBP across $\mathrm{TI}$ within the groups studied were statistically significant, but these changes may not be clinically significant. They may be the result of the effect of sedatives or anaesthetics perturbing physiology in critical illness or the illness itself, such as hypovolemia. Since it was not possible to standardise the medication or intravenous fluid protocols in the participating international centres, variation in use of these with hypotensive side effects can be possible confounders. The recorded SBP after TI in our study suggests 
that the HEMS teams have provided good pre-hospital critical care from scene to hospital admission for these patients. Despite this, one in ten patients in both groups were hypotensive at arrival in hospital. This is a relatively high number and should warrant further investigation to identify if there are reversible causes that can be corrected to improve haemodynamics before arrival in hospital.

\section{Decreased level of consciousness}

Patients with severe TBI and patients with decreased level of consciousness are at high risk of airway obstruction and hypoxia on-scene due to loss of protective airway reflexes and aspiration of blood and gastric contents [14]. Decreased level of consciousness was the main indication for TI in nearly two thirds of the trauma and non-trauma patients in our study, showing that these patients are an important advanced airway indication group in pre-hospital critical care. Competent airway management is vital in preventing secondary insults and improving outcome in trauma and non-trauma patients with decreased level of consciousness [14, 41]. In our study, the rates of hypoxia and hypotension decreased after TI, and there was little difference between the groups studied from TI to hospital admission.

In trauma patients a GCS score below nine is generally considered as an absolute indication for TI, especially in isolated brain injury [42]. In non-trauma patients however, a GCS score cannot be applied in the same way to support the decision to intubate or not [43]. Decreased level of consciousness must therefore be used in a broader context to support decision-making, e.g. when accompanied by persistent hypoxia despite supplemental oxygen administration [42, 43]. However, some trauma and non-trauma patients with higher GCS scores may benefit from prehospital TI to maintain adequate oxygenation and ventilation [44]. A reduction in mortality in patients with GCS below nine receiving pre-hospital physician-led care (instead of paramedic-provided pre-hospital TI) has been shown earlier $[45,46]$. A recent review addressing the effect of pre-hospital TI on mortality in patients with severe TBI found a clear trend towards survival when highly trained providers performed TI compared to providers with limited experience [14]. We found that survival to hospital for patients intubated due to decreased level of consciousness was more favourable than for other indications for TI in physician-staffed HEMS. This effect was strong for non-trauma patients, although they presented with lower mean GCS, higher mean age, and had a higher degree of comobidity than trauma patients.

\section{Survival}

In trauma, there is still a high number of potentially preventable deaths on-scene [47]. Massive haemorrhage and non-compressible haemorrhage are important causes of preventable pre-hospital trauma deaths $[48,49]$. In both trauma and non-trauma patients, there may be patientrelated factors (e.g. cardiopulmonary instability or preexisting comorbidities) that may contribute to lower survival [1]. We have previously shown that pre-hospital TI is safe, with few complications, in the hands of HEMS physicians [24]. In the current study, short-term survival to hospital was not significantly different between trauma and non-trauma patients, and the majority of patients requiring pre-hospital emergency anaesthesia and TI by physician-staffed HEMS presented at the hospital alive.

\section{Limitations}

Although a randomised controlled trial including a control group would have been the preferable standard, this was not feasable in our pre-hospital study setting. The study was therefore designed from a methodological and practical view as a prospective multicenter observational study. Study inclusion was limited to patients who received TI. Those patients for whom TI was attempted but failed and SAD provided were excluded. The data was re-analyzed with these patients included and the results were the same. The strength of this study is the prospective design and the use of a uniform template for data reporting across international HEMS systems. Standardised variables can enhance the quality of data reported, allowing high-quality research data to be compared across patient populations [50]. We believe our results may be generalised to other physician-staffed HEMS. The main limitation was the lack of in-hospital outcome and survival data, which was beyond the scope of this study. Also, the treating physicians recorded the data themselves, with the risk of registration or recall bias. Using anonymous case report forms in this study may have reduced this effect. Automated data capture was not available in the prehospital study setting, and physiological data collected at key intervals according to template definitions may not capture all changes in patient physiology.

\section{Conclusions}

Our results showed that non-trauma patients had a higher incidence of hypoxia before TI than trauma patients, but few were hypoxic at admission. The difference for hypotension was less prominent, but one in ten patients were still hypotensive at admission. Further investigations are needed to identify reversible causes that may be corrected to improve haemodynamics in the pre-hospital setting. We found high survival rates to hospital in both groups, suggesting that physician-staffed HEMS provide high-quality emergency airway management in trauma and non-trauma patients. 


\section{Abbreviations}

ASA-PS: American Society of Anesthesiologists Physical Status; BVM: Bagvalve-mask ventilation; Cl: Confidence interval; cOR: Conditional Odds Ratio; CPAP: Continous positive airway pressure; EMS: Emergency medical services; GCS: Glasgow Coma Score; HEMS: Helicopter Emergency Medical Services; RSI: Rapid sequence intubation; SAD: Supraglottic airway devices; SBP: Systolic blood pressure; $\mathrm{SpO}_{2}$ : Oxygen saturation; TBI: Traumatic brain injury; TI: Tracheal intubation

\section{Acknowledgements}

Assistance with the article: We are grateful to our fellow HEMS physicians for their efforts in submitting patient data in this study. Also, we are indebted to research staff Elizabeth Foster (LAA) and Sandra Ware (GSA-HEMS) for making their service data available. A special thanks to Stig Atle Gjøen for developing and maintaining the database at Haukeland University Hospital. Lastly, our sincere thanks to HEMS colleagues who were instrumental in making this study possible in their home services: Falko Harm, Mathias Zuercher, Péter Temesvári, Andreas Jørgensen, Tom Silfvast, Antti Kämäräinen, Bjørn Ole Reid, Ann-Elin Tomlinson and Lars Jacobsen.

\section{Availability of data and material}

The datasets generated during and/or analysed during the current study are available from the corresponding author on reasonable request.

\section{Funding}

Financial support and sponsorship: A PhD-grant (50\%) for GAS was funded by Norwegian Air Ambulance Foundation, Drøbak, Norway. The participating centres, HEMS physicians and study coordinators received no financial support for their participation in this study.

\section{Authors' contributions}

GAS conceived the study, designed the study protocol, was responsible for ethical applications in Norway, developed and supervised the web-based data collection system, contributed to patient recruitment and data collection, and wrote the first draft and the subsequent manuscript. JKH designed the study protocol, contributed to patient recruitment and data collection, wrote the first draft, and helped draft the manuscript. DL was responsible for ethical applications in UK, contributed to patient recruitment, data collection and helped draft the manuscript. BB was responsible for ethical applications in Australia, contributed to patient recruitment, data collection and helped draft the manuscript. MS contributed to patient recruitment, data collection and helped draft the manuscript. $\mathrm{KOH}$ performed the statistical analyses, prepared the figures and tables, and helped draft the manuscript. JR helped draft the manuscript, and contributed in the interpretation of the statistical analysis. KF contributed to patient recruitment, data collection and helped draft the manuscript. AS was responsible for ethical applications in Hungary, contributed to patient recruitment, data collection and helped draft the manuscript. RL was responsible for ethical applications in UK, contributed to patient recruitment, data collection and helped draft the manuscript. HJ was responsible for ethical applications in Finland, contributed to patient recruitment, data collection and helped draft the manuscript. SJMS conceived the study, designed the study protocol, and helped draft the manuscript. All authors have read and approved the final version of the manuscript.

\section{Ethics approval and consent to participate}

Norway: The Regional Committee for Medical and Health Research Ethics in Western Norway considered the study as a service evaluation study and exempted it from ethical review (Reference number: 2011/1123/REK VEST). Australia: The Royal Prince Alfred Hospital Human Research Ethics Committee approved the study. Hungary: Egészségügyi Tudományos Tanács, Tudományos és Kutatásetikai Bizottság - Scientific and Research Ethics Committee of the Medical Research Council approved the study (Reference number: 98/2012/EKU (3/Pl/12.)). Switzerland: Ethikkommission beider Basel approved the study (Reference number: EK: 233/12). England: The Barts Health R\&D department (for London's Air Ambulance), and the UK National Institute for Health Research and local Ethics and Research Committee (for Kent Surrey Sussex HEMS), approved the study as a service evaluation project and exempted it from ethical review. Finland: The Ethics Committee of Kuopio University Hospital, Kuopio, approved the study (Reference number Kupio / Vantaa / Tampere132/2011, and Tampere ETL code R12020). Requirement for written informed consents was waived.

\section{Consent for publication}

See above. Consent for publication is not applicable.

\section{Competing interests}

The authors declare they have no conflict of interest.

\section{Publisher's Note}

Springer Nature remains neutral with regard to jurisdictional claims in published maps and institutional affiliations.

\section{Author details}

${ }^{1}$ Norwegian Air Ambulance Foundation, Drøbak, Norway. ${ }^{2}$ Department of Anaesthesia and Intensive Care, Haukeland University Hospital, Bergen, Norway. ${ }^{3}$ Department of Health Sciences, University of Stavanger, Stavanger, Norway. ${ }^{4}$ Air Ambulance Department, Oslo University Hospital, Oslo, Norway. ${ }^{5}$ Faculty of Medicine, University of Oslo, Oslo, Norway. ${ }^{6}$ University of Surrey, Guildford, UK. ${ }^{7}$ Kent, Surrey \& Sussex Air Ambulance Trust, Marden, UK. ${ }^{8}$ UiT The Arctic University of Norway, Tromsø, Norway. ${ }^{9}$ The University Hospital of North Norway, Tromsø, Norway. ${ }^{10}$ Sydney HEMS, NSW Ambulance, Sydney, Australia. ${ }^{11}$ Sydney Medical School, University of Sydney, Sydney, Australia.

${ }^{12}$ Centre for Clinical Research, Haukeland University Hospital, Bergen, Norway.

${ }^{13}$ Hungarian Air Ambulance Nonprofit Ltd, Budaors, Hungary. ${ }^{14}$ Centre for Pre-hospital Emergency Care, Kuopio University Hospital, Kuopio, Finland. ${ }^{15}$ London's Air Ambulance, Bartshealth NHS Trust, London, UK. ${ }^{16}$ Department of Medical Sciences, University of Bergen, Bergen, Norway. ${ }^{17}$ Norwegian Air Ambulance Foundation, Møllendalsveien 34, 5009 Bergen, Norway.

Received: 1 February 2017 Accepted: 30 June 2017

Published online: 11 July 2017

\section{References}

1. Hossfeld B, Bein B, Boettiger BW, Bohn A, Fischer M, Graesner JT, Hinkelbein J, Kill C, Lott C, Popp E, et al. Recommended practice for out-of-hospital emergency anaesthesia in adults: statement from the out-of-hospital emergency Anaesthesia working Group of the Emergency Medicine Research Group of the German Society of Anaesthesiology and Intensive Care. Eur J Anaesthesiol. 2016;33(12):881-97.

2. Major trauma. Assessment and initial management [NG39]. London, National Institute for Health and Care Excellence; 2016.

3. Pakkanen T, Virkkunen I, Silfvast T, Randell T, Huhtala H, Yli-Hankala A. One-year outcome after prehospital intubation. Acta Anaesthesiol Scand. 2015;59(4):524-30.

4. Helm M, Hossfeld B, Schafer S, Hoitz J, Lampl L. Factors influencing emergency intubation in the pre-hospital setting-a multicentre study in the German helicopter emergency medical service. Br J Anaesth. 2006;96(1):67-71.

5. Gunning M, O'Loughlin E, Fletcher M, Crilly J, Hooper M, Ellis DY. Emergency intubation: a prospective multicentre descriptive audit in an Australian helicopter emergency medical service. Emerg Med J. 2009;26(1):65-9.

6. Brown CA 3rd, Bair AE, Pallin DJ, Walls RM, Investigators NI. Techniques, success, and adverse events of emergency department adult intubations. Ann Emerg Med. 2015;65(4):363-70. e1

7. Walls RM, Brown CA 3rd, Bair AE, Pallin DJ, Investigators NI. Emergency airway management: a multi-center report of 8937 emergency department intubations. J Emerg Med. 2011;41(4):347-54.

8. Cook T, Behringer EC, Benger J. Airway management outside the operating room: hazardous and incompletely studied. Curr Opin Anaesthesiol. 2012;25(4):461-9.

9. Mort TC. Preoxygenation in critically ill patients requiring emergency tracheal intubation. Crit Care Med. 2005;33(11):2672-5.

10. Shafi S, Gentilello L. Pre-hospital endotracheal intubation and positive pressure ventilation is associated with hypotension and decreased survival in hypovolemic trauma patients: an analysis of the National Trauma Data Bank. J Trauma. 2005;59(5):1140-5. discussion 45-7

11. Funk DJ, Jacobsohn E, Kumar A. Role of the venous return in critical illness and shock: part II-shock and mechanical ventilation. Crit Care Med. 2013;41(2):573-9.

12. Den Hartog D, Romeo J, Ringburg AN, Verhofstad MH, Van Lieshout EM. Survival benefit of physician-staffed helicopter emergency medical services (HEMS) assistance for severely injured patients. Injury. 2015;46(7):1281-6. 
13. Lockey D. Improving UK trauma care: the NCEPOD trauma report. Anaesthesia. 2008:63(5):455-7.

14. Bossers SM, Schwarte LA, Loer SA, Twisk JW, Boer C, Schober P. Experience in Prehospital Endotracheal intubation significantly influences mortality of patients with severe traumatic brain injury: a systematic review and metaanalysis. PLoS One. 2015;10(10):e0141034.

15. Chesnut RM, Marshall LF, Klauber MR, Blunt BA, Baldwin N, Eisenberg HM, Jane JA, Marmarou A, Foulkes MA. The role of secondary brain injury in determining outcome from severe head injury. J Trauma. 1993:34(2):216-22.

16. Holler JG, Bech CN, Henriksen DP, Mikkelsen S, Pedersen C, Lassen AT. Nontraumatic hypotension and shock in the emergency department and the prehospital setting, prevalence, etiology, and mortality: a systematic review. PLoS One. 2015;10(3):e0119331.

17. Jones AE, Stiell IG, Nesbitt LP, Spaite DW, Hasan N, Watts BA, Kline JA. Nontraumatic out-of-hospital hypotension predicts inhospital mortality. Ann Emerg Med. 2004:43(1):106-13.

18. Lockey D, Crewdson K, Weaver A, Davies G. Observational study of the success rates of intubation and failed intubation airway rescue techniques in 7256 attempted intubations of trauma patients by pre-hospital physicians. Br J Anaesth. 2014;113(2):220-5.

19. Lossius HM, Sollid SJ, Rehn M, Lockey DJ. Revisiting the value of pre-hospital tracheal intubation: an all time systematic literature review extracting the Utstein airway core variables. Crit Care. 2011;15(1):R26.

20. Davis DP. The need for standardized data reporting for prehospital airway management. Crit Care. 2011;15(2):133.

21. Wigman LD, van Lieshout EM, de Ronde G, Patka P, Schipper IB. Traumarelated dispatch criteria for helicopter emergency medical Services in Europe. Injury. 2011;42(5):525-33.

22. Helm M, Kremers G, Lampl L, Hossfeld B. Incidence of transient hypoxia during pre-hospital rapid sequence intubation by anaesthesiologists. Acta Anaesthesiol Scand. 2013;57(2):199-205.

23. Sollid SJ, Lockey D, Lossius HM. Pre-hospital advanced airway management expert g: a consensus-based template for uniform reporting of data from pre-hospital advanced airway management. Scand J Trauma Resusc Emerg Med. 2009:17:58.

24. Sunde GA, Heltne JK, Lockey D, Burns B, Sandberg M, Fredriksen K, Hufthammer KO, Soti A, Lyon R, Jantti $H$, et al. Airway management by physician-staffed helicopter emergency medical services - a prospective, multicentre, observational study of 2,327 patients. Scand J Trauma Resusc Emerg Med. 2015;23:57.

25. Localio AR, Berlin JA, Ten Have TR, Kimmel SE. Adjustments for center in multicenter studies: an overview. Ann Intern Med. 2001;135(2):112-23.

26. R Core Team. R: a language and environment for statistical computing. In: Vienna: R foundation for statistical computing; 2015.

27. Merz TM, Etter R, Mende L, Barthelmes D, Wiegand J, Martinolli L, Takala J. Risk assessment in the first fifteen minutes: a prospective cohort study of a simple physiological scoring system in the emergency department. Crit Care. 2011;15(1):R25.

28. Davis DP, Dunford JV, Poste JC, Ochs M, Holbrook T, Fortlage D, Size MJ, Kennedy F, Hoyt DB. The impact of hypoxia and hyperventilation on outcome after paramedic rapid sequence intubation of severely headinjured patients. J Trauma. 2004;57(1):1-8. discussion 8-10

29. Davis DP, Fisher R, Buono C, Brainard C, Smith S, Ochs G, Poste JC, Dunford JV. Predictors of intubation success and therapeutic value of paramedic airway management in a large, urban EMS system. Prehosp Emerg Care. 2006:10(3):356-62.

30. Seymour CW, Cooke CR, Heckbert SR, Copass MK, Yealy DM, Spertus JA, Rea TD. Prehospital systolic blood pressure thresholds: a community-based outcomes study. Acad Emerg Med. 2013;20(6):597-604.

31. Vincent JL, De Backer D. Circulatory shock. N Engl J Med. 2013;369(18):1726-34.

32. Jensen AG, Callesen T, Hagemo JS, Hreinsson K, Lund V, Nordmark J, Clinical Practice Committee of the Scandinavian Society of A, Intensive Care M. Scandinavian clinical practice guidelines on general anaesthesia for emergency situations. Acta Anaesthesiol Scand. 2010;54(8):922-50.

33. Nakstad AR, Heimdal HJ, Strand T, Sandberg M. Incidence of desaturation during prehospital rapid sequence intubation in a physician-based helicopter emergency service. Am J Emerg Med. 2011;29(6):639-44.

34. Rognas L, Hansen TM, Kirkegaard H, Tonnesen E. Pre-hospital advanced airway management by experienced anaesthesiologists: a prospective descriptive study. Scand J Trauma Resusc Emerg Med. 2013;21(1):58.
35. Wimalasena Y, Burns B, Reid C, Ware S, Habig K. Apneic oxygenation was associated with decreased desaturation rates during rapid sequence intubation by an Australian helicopter emergency medicine service. Ann Emerg Med. 2015;65(4):371-6.

36. Soti A, Temesvari P, Hetzman L, Eross A, Petroczy A. Implementing new advanced airway management standards in the Hungarian physician staffed helicopter emergency medical service. Scand I Trauma Resusc Emerg Med. 2015;23(1):3.

37. Peters J, Bruijstens L, van der Ploeg J, Tan E, Hoogerwerf N, Edwards M. Indications and results of emergency surgical airways performed by a physician-staffed helicopter emergency service. Injury. 2015;46(5):787-90.

38. Poloujadoff MP, Lapostolle F, Lockey D, Amathieu R, Merouani M, Galinski M, Adnet F. Survival of severely shocked patients who present with absent radial pulse and unrecordable blood pressure in the pre-hospital phase. Resuscitation. 2006:69(2):185-9.

39. Spaite DW, Hu C, Bobrow BJ, Chikani V, Sherrill D, Barnhart B, Gaither JB, Denninghoff KR, Viscusi C, Mullins T, et al. Mortality and Prehospital blood pressure in patients with major traumatic brain injury: implications for the hypotension threshold. JAMA surgery. 2017;152(4):360-8.

40. Dellinger RP, Levy MM, Rhodes A, Annane D, Gerlach H, Opal SM, Sevransky $J E$, Sprung $C L$, Douglas IS, Jaeschke R, et al. Surviving sepsis campaign: international guidelines for management of severe sepsis and septic shock: 2012. Crit Care Med. 2013;41(2):580-637.

41. Boer C, Franschman G, Loer SA. Prehospital management of severe traumatic brain injury: concepts and ongoing controversies. Curr Opin Anaesthesiol. 2012;25(5):556-62.

42. Winchell RJ, Hoyt DB. Endotracheal intubation in the field improves survival in patients with severe head injury. Trauma research and education foundation of san Diego. Arch Surg. 1997;132(6):592-7.

43. Nielsen K, Hansen CM, Rasmussen LS. Airway management in unconscious non-trauma patients. Emerg Med J. 2012;29(11):887-9.

44. Ellis DY, Davies GE, Pearn J, Lockey D. Prehospital rapid-sequence intubation of patients with trauma with a Glasgow coma score of 13 or 14 and the subsequent incidence of intracranial pathology. Emerg Med J. 2007;24(2):139-41.

45. Murray JA, Demetriades D, Berne TV, Stratton SJ, Cryer HG, Bongard F, Fleming A, Gaspard D. Prehospital intubation in patients with severe head injury. J Trauma. 2000;49(6):1065-70.

46. Garner AA, Mann KP, Fearnside M, Poynter E, Gebski V. The head injury retrieval trial (HIRT): a single-centre randomised controlled trial of physician prehospital management of severe blunt head injury compared with management by paramedics only. Emerg Med J. 2015;32(11):869-75.

47. Oliver GJ, Walter DP, Redmond AD. Are prehospital deaths from trauma and accidental injury preventable? A direct historical comparison to assess what has changed in two decades. Injury. 2017:48(5):978-84.

48. Glen J, Constanti M, Brohi K, Guideline Development G. Assessment and initial management of major trauma: summary of NICE guidance. BMJ. 2016;353:13051

49. Stanworth SJ, Davenport R, Curry N, Seeney F, Eaglestone S, Edwards A, Martin K, Allard S, Woodford M, Lecky FE, et al. Mortality from trauma haemorrhage and opportunities for improvement in transfusion practice. $\mathrm{Br}$ J Surg. 2016;103(4):357-65.

50. Lossius HM, Kruger AJ, Ringdal KG, Sollid SJ, Lockey DJ. Developing templates for uniform data documentation and reporting in critical care using a modified nominal group technique. Scand J Trauma Resusc Emerg Med. 2013:21:80 